\title{
The source of and a simple way to avoid ground loops in biasing Langmuir probe array
}

\author{
I. Shesterikov, ${ }^{a, 1}$ K. Crombe, ${ }^{b, c}$ R. Ochoukov, ${ }^{a}$ A. Kostic,,${ }^{a, b}$ M. Usoltceva, ${ }^{a, b}$ and J.-M. \\ Noterdaeme, ${ }^{a, b}$ \\ ${ }^{a}$ Max-Planck-Institut für Plasmaphysik, \\ D-85748 Garching, Germany \\ ${ }^{b}$ Ghent University, Department of Applied Physics, \\ B-9000 Gent, Belgium \\ ${ }^{c} L P P-E R M / K M S$, \\ B-1000 Brussels, Belgium \\ E-mail: ilys@ipp.mpg.de
}

\begin{abstract}
Biasing multiple Langmuir probes arranged in an array through one voltage source presents a more compact, reliable and convenient way to bias than using an individual source for each probe. This approach however is the subject of ground loops and several methods have been discussed to avoid them. The high frequency isolation transformer is considered to be a best way to break ground loops since it does not require an additional power supplies and, in general, any other electric circuit. The presented results demonstrate a considerable suppression of the ground loop noise using this technique. The following limitations should be considered in this approach: only an AC voltage bias should be applied, the relatively high internal resistance of the transformer should be taken into account, the frequency of the AC bias signal should correspond to the transforner bandwidth.
\end{abstract}

KeYwords: Plasma diagnostics - probes, Plasma generation (laser-produced, RF, x ray-produced)

\footnotetext{
${ }^{1}$ Corresponding author.
} 


\section{Contents}

1 Introduction 1

2 Biasing Langmuir probe array 1

3 Solutions 2

4 Conclusion 6

\section{Introduction}

Langmuir probes are one of the most common diagnostic tools to characterize a low temperature plasma [1,2]. Although it is considered to be a perturbing diagnostic, its advantages overweight this drawback. Probes provide a complete set of general plasma parameters such as electron temperature $T_{e}$, electron density $n_{e}$ and plasma potential $U_{p}$ and, taking into account its simplicity, occupy an extensive niche in plasma diagnostics. To get a spatial distribution of plasma parameters a probe can either be installed on a movable manipulator system or multiple probes can be arranged in an array. The array configuration provides simultaneous measurements from multiple spatial points and thus significantly reduces the required experimental time. Moreover, simultaneous measurements from all spatial points are especially important for poorly reproducible discharges, such for instance arc discharges, when the spatial plasma profile (i.e. the arc position) unpredictably changes from discharge to discharge.

\section{Biasing Langmuir probe array}

One common problem in designing such an array is the design of the circuit topology to bias all probes and to measure the corresponding currents and voltages on the probes. The three key elements of the probe bias and measurements system are (i) the bias voltage generator, (ii) the probe unit and (iii) the data acquisition system. The purpose of the probe unit is to measure the voltage applied to the probe pin and the current flowing through a probe. These two signals constitute two output signals of the probe unit, which are recorded by the data acquisition system. The design details of the probe unit falls outside the scope of this work. A certain difficulty is related to the development of an optimal topology to bias multiple probes arranged as a Langmuir probe array. If all probes are decoupled from each other and biased independently using separate voltage sources consisting of multiple signal generators/amplifier for each probe, such as in Fig. 1(a), than the system could be very bulky, expensive and unreliable. Moreover, since the voltage bias on all probes are uncorrelated, one needs then to acquire current and voltage signals from each probe, which could be challenging or expensive Âă in case of big arrays due to the lack of data acquisition channels. It is more effective to bias all probes through one source by connecting all probes in 
parallel, as it is shown in Fig. 1(b). Bias signals in this case are identical on all probes and it suffices to acquire only the current signals from the probe array and one single voltage signal. In this way the number of required data acquisition channels is strongly reduced. However, such a design has a conceptual problem: the ground lines of different current and voltage measurement units are connected in two points creating a ground loop. One point is the ground line of the bias voltage source. Another point is the ground line of the metal chassis of the data acquisition system. Ground loop currents can be induced by stray AC magnetic fields (B, green arrows). The ground loop constitutes a conductive wire loop, which may have a large area of several square meters. It acts like a short-circuited single-turn "transformer winding". Any AC magnetic flux passing through the loop, from power supply transformers or just adjacent power cables will induce currents in the loop by induction. Since its resistance is very low, frequently less than $1 \mathrm{Ohm}$, the induced currents can be large. As a result, it causes a significant noise in the signal lines making measurements impossible. Moreover, other measurement channels are connected together in parallel, enhancing the noise level on each signal line, since every cable interconnects with all other cables forming multiple paths to the ground.

Figures 2(a)-(e) show results from a simple test to demonstrate how the noise level depends on the number of used channels. Results (a)-(d) show probe array signals from different channels measured without plasma such that the contribution of the ground loop noise is clearly visible. The curve in Fig.2(a) shows results from only one channel, which corresponds to a ground loop free result. As the number of connected units is gradually increased in Figures2(a)-(d), the noise level in each channel also changes. Apparently the more channels the larger the noise level, which makes the implementation of this concept for large probe arrays impractical. The typical power spectrum of such a noise is shown in Fig.2(e). It demonstrates that spurious ground loop current creates noise at the $\mathrm{AC}$ mains base frequency $(50 \mathrm{~Hz})$ and at the harmonics thereof $(150 \mathrm{~Hz}, 250 \mathrm{~Hz}$ and so on) confirming that a ground loop current is induced by stray AC magnetic fields from adjacent power cables or transformers.

\section{Solutions}

There are several solutions to ground loop noise all of which are based on breaking the ground loop or, in other words, prevent the current from flowing. One method is to galvanically decouple current and voltage signals from the data acquisition system using so called "isolation amplifier", such for instance as "ISO124". Such an amplifier should then be installed between the probe unit and data acquisition system since it is developed only to transfer the measured signal and not the power. Another way is to digitize current and voltage signals right at the exit from the probe unit, transfer them optically and convert them back to the analog form before acquiring in the data acquisition system. While the first method is relatively simple to implement, it still requires separate power sources (also decoupled from the ground) to power the isolation amplifier and associated circuit. Meanwhile, the corresponding application circuit must be developed. The second method can be implemented only using commercially available converters and, taking into account the large number of probes in array, it is not very practical.

An isolation transformer is considered to be the best way to break ground loops[3, 4]. It is simple to implement, compact, not expensive, and does not require additional power supplies or 
(a)

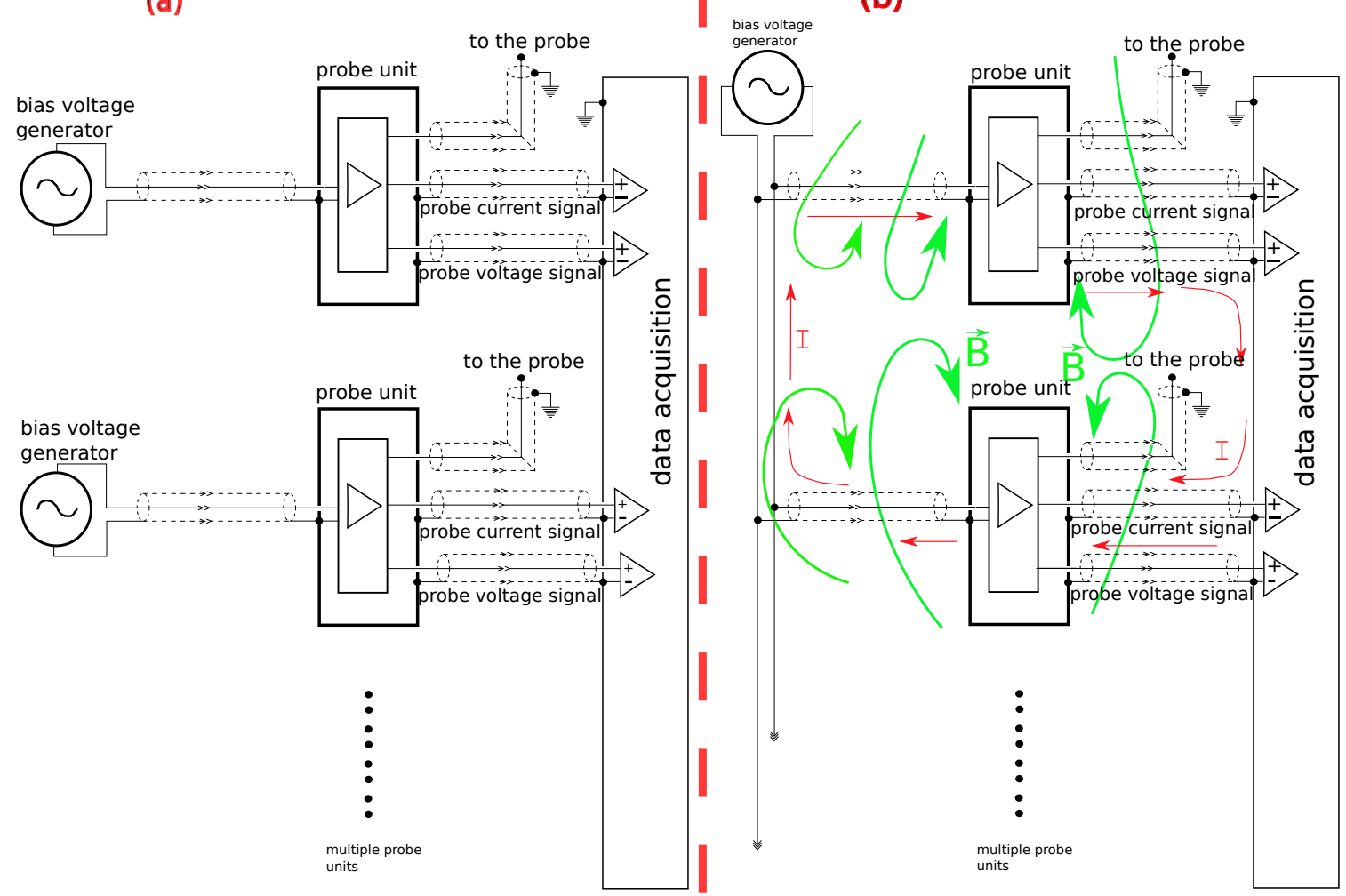

Figure 1. Schematic view of a Langmuir probe array bias circuit. (a) Each probe in an array has an individual voltage bias source. Such a topology is free of ground loops, but requires a number of voltage generators, which makes it bulky and expensive. Meanwhile, voltage AC signals from generators are uncorrelated, which requires two data acquisition channels for each probe (one for voltage and one for current). (b) Biasing all probes through one bias source. All probes are connected in parallel to one voltage generator. The proposed circuit is compact and, since voltage signals are identical for all probes, it requires half as many data acquisition channels as in circuit (a). Such a topology however is subject to ground loops. The ground loop currents (red arrows) are induced by the stray magnetic field (green arrows) from the surrounding equipment and power cables.

electric circuits in general. This passes AC signal, power, and keeps everything grounded, but will not pass ground loop currents at all, as it breaks the DC connection between components. In Fig. 2(f) the transformers are installed between voltage generator and probe units. Although transformer installed between the probe units and data acquisition system will also break the ground loop, one should take into account that the output current signal of the probe unit is non-harmonic since the Langmuir probe current-voltage (IV) characteristics are nonlinear. As a consequence, the current response to the harmonic voltage bias signal contains a significant DC component, which will be shortened by the transformer primary winding. This in turn could lead to a malfunction of the probe unit output channel. Figure 3 shows a reduction in the noise level using an isolation transformer. Figures (a) and (b) compare signals from several channels measured without (a) and with (b) isolation transformers. Corresponding circuit topologies are shown in Fig. 1(b) and Fig. 2(f), respectively. One can notice that the noise level in Fig. 3(a) is comparable to the ion 

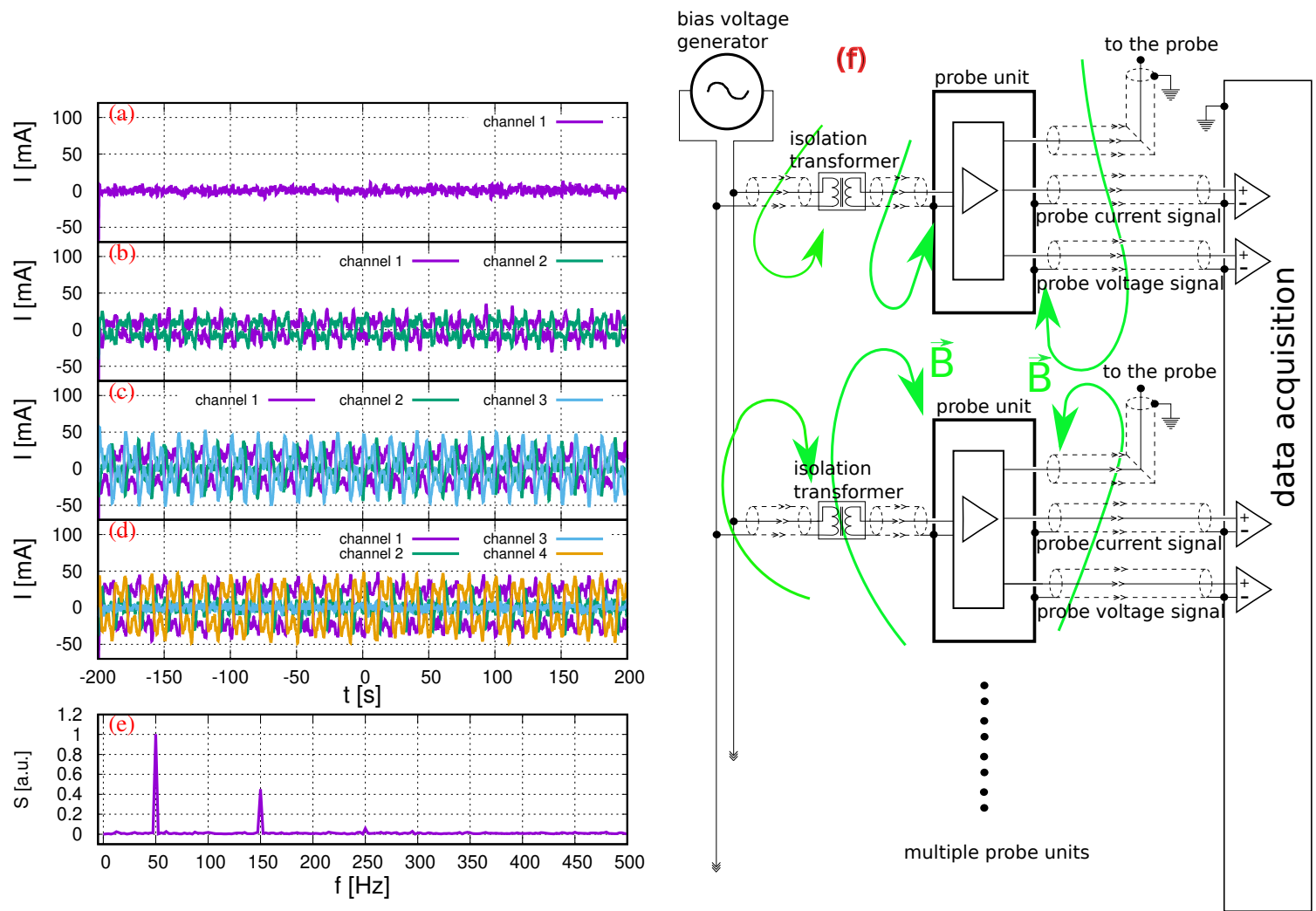

Figure 2. Creating ground loops following the circuit topology as in Fig. 1(b). (a) Only one channel, which corresponds to a ground loop free and, correspondingly, not noisy result. (b)-(d) Rising the number of used channels creates more ground loops, which directly influence the noise level on all measured channels. This makes the implementation of this concept for large probe arrays impractical. (e) The typical power spectrum of a ground loop noise. The adjacent power transformers or power cables induce AC magnetic flux and corresponding ground loop current at the $\mathrm{AC}$ main base frequency $(50 \mathrm{~Hz})$ and the harmonics. (f) Break in the ground loop current path using isolation transformers. The transformers are installed between the generator and the probe units and are connected in parallel and powered through one voltage generator. The transformer breaks the induced common ground loop current while passing the $\mathrm{AC}$ bias voltage and power from the generator.

current thus complicating the estimation of the ion saturation current, floating potential and electron temperature. The ground loop free measurements in Fig. 3(b) show a considerably more accurate measurement illustrating the beneficial role of the transformer.

Several limitations of eliminating ground loop using isolation transformer need to be taken into account. First, one should bias the probe only by the AC signal. The AC bias however frequently is the most interesting for the measurements: in order to get plasma density $n_{e}$ and electron temperature $T_{e}$ it is essential to measure the entire probe characteristic and not simply the ion saturation current. This is especially true if the measured plasma characteristics are in radio-frequency (RF) produced plasmas. Indeed, strong RF electric field oscillations lead to strong oscillations of plasma potential, the so-called sheath rectification effect. In this case, a characteristic of the probe averaged over one RF cycle differs significantly from an instantaneous characteristic. A variety of techniques have 

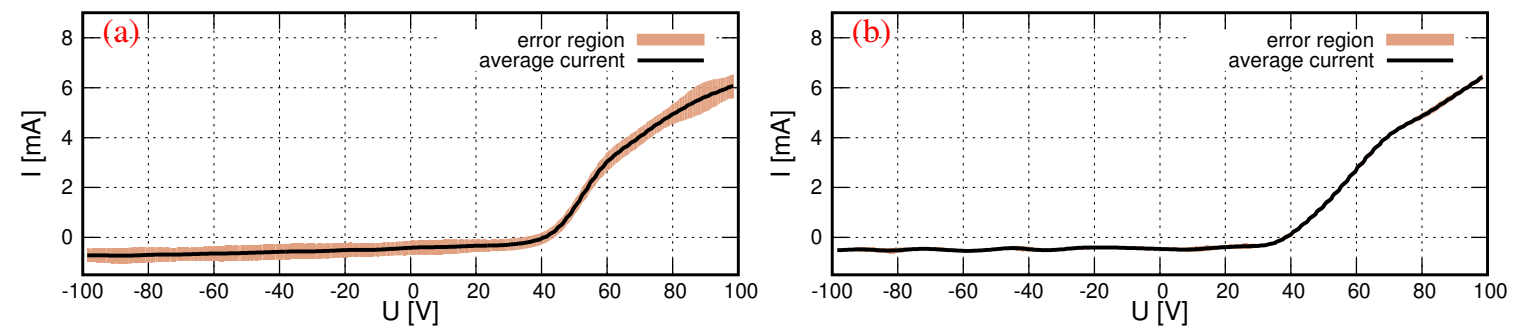

Figure 3. Effect of the ground loop break on the measured probe IV characteristics. The results in figure (a) correspond to the IV characteristic measured with multiple ground loops. The error-bar is comparable to the level of an ion-saturation current, complicating an estimation of the plasma density. Isolation transformers were used for the results in figure (b) with less noisy results.

been developed to extract data from probes in an RF environment [2, 5]. However, one needs to be sure that the applied technique works properly and the measured characteristics are not altered by the sheath rectification. For that reason one always needs to measure the entire probe characteristic using sweeping technique.

The second limitation is that the isolation transformer has a relatively high parasitic resistance (from several Ohm to several hundreds of Ohm). This could be a problem when one collects a large electron current in a dense plasma, when the effective sheath resistance is comparable to the transformer resistance. Note that the collection of an electron saturation current is inevitable when one uses a sweeping technique because the sweeping voltage range should be set high enough to measure the plasma potential and in this case one inevitably pulls a relatively high electron saturation current. Too high current through the transformer could be noticed as a reduction of the sweeping voltage amplitude at the transformer output since the transformer output and load both form a simple voltage divider network. The simplest way to avoid this is to reduce the probe collecting area. Alternatively, one can install an intermediate push-pull amplifier circuit [6] between the transformer output and the probe. However, although it is in principle doable, this solution levels the simplicity of the proposed decoupling method.

Another feature that one needs to take into account is the transformer bandwidth [7]. Depending on the specific application, the transformer can be developed only for a certain frequency range. If the sweeping frequency needs to be varied in a relatively wide range up to hundreds of $\mathrm{kHz}$ then it is recommended to use a conventional audio transformer, which could have a bandwidth from several $\mathrm{Hz}$ to several hundreds of $\mathrm{kHz}$. For instance one can consider the audio transformer model "CUI30A04E" (trademark "OEP") which has been used in this work. It has a maximal operation frequency of $20 \mathrm{kHz}$ and an applied $\mathrm{AC}$ voltage amplitude could be up to $500 \mathrm{~V}$ with the power rating of $2 \mathrm{~W}$. If the high frequency and high bandwidth are not an issue, one could use a simple cheap printed circuit board (PCB) transformer, such for instance as "FL 14/6" from ${ }^{\circledR}$ Block. Although they are developed to operate merely at a principle frequency of about $50 \mathrm{~Hz}$, they can be used up to several tens of $\mathrm{kHz}$. 


\section{Conclusion}

Simultaneous biasing multiple Langmuir probes from one voltage source is subject to ground loops. The induced ground currents cause significant noise in the signal lines, which may significantly affect the measured IV characteristic of the probe. An isolation transformer presents a simple and cheap solution to break the ground loop. Moreover it does not require additional power supplies.. This approach, however, has its own limitations. First, only AC bias can be applied. Second, one should take into account the internal resistance of the transformer, which should be negligible comparing to the sheath resistance of the Langmuir probe. This, however, is not always the case especially in a dense plasma and for a large probe collecting area. The transformer frequency bandwidth sets another limitation. Authors recommend using conventional audio transformers which could have a bandwidth up to several hundreds of $\mathrm{kHz}$.

\section{Acknowledgments}

This work has been carried out within the framework of the EUROfusion Consortium and has received funding from the Euratom research and training programme. 2014-2018 under grant agreement No 633053. The views and opinions expressed herein do not necessarily reflect those of the European Commission.

\section{References}

[1] Hutchinson I. H. Principles of Plasma Diagnostics 2009: Cambridge University Press

[2] Hershkowitz N. How Langmuir probe works in Plasma Diagnostics: Discharge Parameters and Chemistry, Band 1 1989: Academic Press

[3] Ott Henry W. Noise Reduction Techniques in Electronic Systems 1988: Wiley \& Sons. pp. 1194-1217

[4] Horowitz P. and Hill W. The Art of Electronics. Third edition 2015: Cambridge University Press. pp. 583-589

[5] Sudit, I.D. and Chen, F.F Plasma Sources Sci. Technol. 3, 162-168, 1994

[6] Tietze U., Schenk Ch., Gamm E. Electronic Circuits. Handbook for Design and Application 1991: Springer, Heidelberg

[7] Glen M. Ballou. Handbook for Sound Engineers. Fourth Edition 1998: Elsevier 